\title{
A Network Theory of Nonlinear Viscoelasticity
}

\author{
Shizuo HAYASHI \\ Department of Physics, Faculty of General Study, Gunma \\ University, Aramaki-cho, Maebashi 371, Japan.
}

(Received July 5, 1977)

\begin{abstract}
Two kinds of relaxation processes are introduced for the theory of nonlinear viscoelasticity of temporarily crosslinked network structure. One is the chainslip and the other is the change in number of chains as a function of time. Three assumptions are made for this theory: (1) The relaxation process for the chain-slip is a linear process characterized by a single relaxation time; (2) The chain breakage coefficient is proportional to the absolute value of the average force acting on a chain; (3) The rate of chain creation is constant. The following results are obtained for typical deformations. (1) The viscosity in the steady flow with a constant shear rate is non-Newtonian and at high shear rates its molecular weight dependence deviates from the 3, 4-th power law of viscosity. (2) The stress growth at the onset of the shear flow with a constant shear rate shows a stress overshoot which is prominent at a high shear rate. (3) The stress relaxation at a constant deformation shows a non-linear strain dependence corresponding to relatively short periods of time and a linear relaxation tendency for relatively long periods of time. (4) The stress relaxation at a sudden termination of the shear flow shows a strain rate dependence initially and a linear tendency for long periods of time. A new type of constitutive equation in which the memory function is one of the invariants of the internal strain determined by the slip equation was found by investing the relation between the present theory and the phenomenological constitutive equation.

KEY WORDS Nonlinear Viscoelasticity / Network Structure / Stress Overshoot / Stress Relaxation / Constitutive Equation /
\end{abstract}

Viscoelastic behaviors of a polymer melts and concentrated polymer solutions are generally nonlinear and the experiments to meet the linearity condition for these systems are not easy. Whether the conditions for linearity are being satisfied or not must be determined in the experiments for linear viscoelastic phenomena. When we consider this, it is quite natural to expect that nonlinear viscoelastic phenomena may be the use of the nonlinear theory. At this stage, it is important to find some constitutive equations to explain consistently the nonlinear viscoelastic phenomena.

One of the theoretical approaches to the viscoelasticity of polymer melts and concentrated polymer solutions is the network theory based on a temporarily crosslinked network model. The network theory is classified into two types. One is the chain breakage-and-reformation theory ${ }^{1-3}$ in which the relaxation processes are originated in the chain breakage and chain reformation, and the other is the chain slip theory ${ }^{4-6}$ which by the chain slip controls the relaxation processes. Both of these give the same formalism within the limits of the linear theory. ${ }^{4}$ One of the slip theories is extended to the nonlinear theory. ${ }^{6}$ This is applicable only to the steady laminar flow but not to the general viscoelastic phenomena. Yamamoto's chain-breakage-and-reformation theory has the most general formalism at this stage and it substantially covers the nonlinear theory. At 


\section{S. HAYASHI}

the time Yamamoto's theory was presented, however, the nonlinear viscoelastic phenomena had not yet been studied well experimentally and thus the theory had not been applied to practical problems for a long time. Recently, Takano applied Yamamoto's theory to certain practical problems and succeeded in explaining the stress overshoot phenomena and other viscoelastic properties of concentrated polymer solutions and melts. ${ }^{7}$

The other theoretical approach is the study of phenomenological constitutive equations. Many authors have presented various constitutive equations, ${ }^{1,8-22}$ and investigated their applicability. $^{22-26}$ The phnomenological theory has the advantage of being applicable to the general features of nonlinear viscoelastic properties, since it is not based on a definite molecular model of the system considered. The constitutive equations have generally the integral formula because of their ease of application to practical problems and they are principally included in Lodge's equation ${ }^{8}$ whose presentation is based on the network theory. Although the constitutive equations presented up till now have successfully explained the nonlinear viscoelastic properties, all of them have weak points. That is, we can explain some aspects of viscoelastic properties by one constitutive equation but not any others. The strain dependent constitutive equation, containing the invariant of the strain tensor in its memory function, seems to have broad application, though considerable tedious calculation is involved for the analysis of the experimental results. $^{22}$ Therefore, one important problem we have is to find out a constitutive equation which is applicable to the whole nonlinear viscoelastic phenomena.

At this point the phenomenological theory has not yet been well established, and we are not sure whether presentation of molecular theory and the nonlinear network theory are meaningful or not. Nevertheless, the molecular theory will have enough meaning to enable us to interpret the nonlinear viscoelastic phenomena in molecular terms, or directly in terms of the change in the number of chains of network structure.

We should now like to consider a crosslinked network structure. When deformed, the rates of chain breakage and chain reformation are different from those in the stress free state. Yamamoto dealt with these effects in his theory. But we should like to consider these effects in a different way. The "chain" in our treatment refers to that part of the polymer molecule between the two adjacent cross-linkages and whole polymer molecule is termed the "molecular chain." In the deformed state, the molecular chains in the network slip from one to another in an entangled state and the terminal chain in each molecular chain slips out of the junction point and is lost. Afterward the terminal chain in the molecular chain will be recrosslinked and the chain reformed. Accordingly, the average deformation of chains will be smaller than the deformation given for the system. In the chain breakage-and-reformation processes both the chain slip and the net change in the number of chains induces the structural change of network structure. A consideration of these two effects gives rise to nonlinearity which we already talked about in one of our previous reports, ${ }^{5}$ but this was not applied to practical problems. In this article we present a revised treatment and show its application to some practical problems.

\section{THEORY}

Let the end-to-end vector of the $i$ th chain of a molecular chain in the network be $\boldsymbol{r}_{i}$, and the strain free energy be $\phi\left(\left|\boldsymbol{r}_{i}\right|\right)$, the stress is given thus as

$$
\boldsymbol{\sigma}=\sum_{P} \sum_{i}\left(\frac{1}{r_{i}} \frac{\mathrm{d} \phi}{\mathrm{d} r_{i}}\right)\left(\boldsymbol{r}_{i} \boldsymbol{r}_{i}\right)-P \mathbf{1}
$$

where $\sum_{i}$ is the summation with respect to the number of chains in a molecular chain, $\sum_{P}$, the number of molecular chains in unit volume of the network, $\left(\boldsymbol{r}_{i} \boldsymbol{r}_{i}\right)$ the dyadic, $P$ the internal (hydrostatic) pressure and 1 the unit tensor.

In order to simplify the treatment we assume

(1) The slip process is characterized by a single relaxation time.

The internal strain of the chains is characterized by the slip equation containing a single relaxation time, so that chains have a common equal internal strain if they are Gaussian. In this case the strain free energy of the $i$ th chain 
is given as

$$
\left.\begin{array}{c}
\dot{\phi}\left(\left|\boldsymbol{r}_{i}\right|\right)=\frac{3 k_{\mathrm{B}} T}{2 l_{i}^{2}} \boldsymbol{r}_{i}^{2} \\
\left(\boldsymbol{r}_{i} \boldsymbol{r}_{i}\right)=\frac{l_{i}^{2}}{3} \boldsymbol{\alpha}
\end{array}\right\}
$$

where $l_{i}$ is the mean square end-to-end distance of the chain; $\boldsymbol{\alpha}$, the internal strain of the chain, $k_{\mathrm{B}}$, the Boltzmann's constant and $T$, the absolute temperature. The double summation in eq 1 is taken with respect to the number of chains $\nu$ in unit volume of the network structure, so that, we have

$$
\boldsymbol{\sigma}(t)=\nu k_{\mathrm{B}} T \boldsymbol{\alpha}(t)-P \boldsymbol{I}
$$

Let the single relaxation time for the slip process of the chains be $\tau_{0}$ and the deformation tensor be $\boldsymbol{\gamma}(t)$, the slip equation is thus expressed as ${ }^{5}$

$$
\dot{\gamma} \cdot \boldsymbol{\gamma}^{-1} \cdot \boldsymbol{\alpha}+\boldsymbol{\alpha} \cdot \boldsymbol{\gamma}^{+-1} \cdot \dot{\boldsymbol{\gamma}}+\dot{\boldsymbol{\alpha}}=\frac{1}{\tau_{0}}(\boldsymbol{\alpha}-\boldsymbol{I})
$$

where the dot means the time derivative and the dagger, the transpose of the tensor.

If the chain breakage coefficient be $k$ and the rate of chain creation be $g$, then the rate of change in number of chains is expressed as

$$
\frac{\mathrm{d} \nu}{\mathrm{d} t}=-k \nu+g
$$

In the stress free state $\mathrm{d} \nu / \mathrm{d} t=0$, and the relation

$$
k_{e} \nu_{e}=g_{e}
$$

is satisfied, and the suffix $e$ are the quantities in the stress free state or equilibrium state. In a deformed state, $k$ and $g$ may generally be functions of the invariants of the strain tensor and the strain rate tensor, but we make the following assumptions:

(2) The chain breakage coefficient is proportional to the absolute value of the average force acting on a chain.

Let the end-to-end vector of a chain in the stress free state be $\boldsymbol{r}_{0}$, and that in the deformed state be $r$; the average square of end-to-end distance $\left\langle\boldsymbol{r}^{2}\right\rangle$ of a chain in the network may thus be given as;

$$
\left\langle\boldsymbol{r}^{2}\right\rangle=a^{+} \cdot\left\langle\left(\boldsymbol{r}_{0} \boldsymbol{r}_{0}\right)\right\rangle \cdot a=\frac{1}{3} \operatorname{Tr} \boldsymbol{a}^{+} \cdot a\left\langle r_{0}^{2}\right\rangle
$$

where $\boldsymbol{a}$ is the deformation tensor of chains, and \langle\rangle the average with respect to the network. The magnitude of the average force acting on the end of a chain is thus proportional to $\sqrt{\left\langle\boldsymbol{r}^{2}\right\rangle}$; that is $\left(\operatorname{Tr} \boldsymbol{a}^{+} \cdot \boldsymbol{a}\right)^{1 / 2}$.

On the other hand, the internal strain $\boldsymbol{\alpha}$ in eq 1 is the average strain tensor of the chains and is equal to $\boldsymbol{a} \cdot \boldsymbol{a}^{+}$; thus

$$
\boldsymbol{\alpha}=\boldsymbol{a} \cdot \boldsymbol{a}^{+}
$$

Therefore, from assumption 2, we obtain

$$
k=k_{e}\left(\begin{array}{c}
\operatorname{Tr} \boldsymbol{\alpha} \\
3
\end{array}\right)^{1 / 2}
$$

since $\operatorname{Tr} \boldsymbol{a}^{+} \cdot \boldsymbol{a}=\operatorname{Tr} \boldsymbol{a} \cdot \boldsymbol{a}^{+}$and the chain breakage coefficient in the stress free state is $k_{e}$.

Assumption 3: The rate of the chain generation is constant. That is,

$$
g=g_{e}=k_{e} \nu_{e}
$$

This assumption used facilitates the calculation.

The content of this theory is set forthfully in the two equations 4 and 5 with additional condition in eq 9.

The solution of eq 4 is given as

$$
\begin{gathered}
\alpha(t)=\int_{-\infty}^{t} \frac{\mathrm{e}^{-\left(t-t^{\prime}\right) / \tau_{0}}}{\tau_{0}} \gamma\left(t, t^{\prime}\right) \cdot \gamma^{+}\left(t, t^{\prime}\right) \mathrm{d} t^{\prime} \\
\gamma\left(t, t^{\prime}\right)=\gamma(t) \cdot \gamma^{-1}\left(t^{\prime}\right)
\end{gathered}
$$

where $\gamma\left(t, t^{\prime}\right)$ is the relative deformation tensor at the time $t$ with respect to the previous time $t^{\prime}$.

Integration of eq 5 gives

$$
\nu(t)=\mathrm{e}^{-\int_{0}^{t} k\left(t^{\prime}\right) \mathrm{d} t^{\prime}}\left[\nu(0)+\int_{0}^{t} g \mathrm{e}^{\int_{0}^{t^{\prime} k\left(t^{\prime \prime}\right) \mathrm{d} t^{\prime \prime}}} \mathrm{d} t^{\prime}\right]
$$

and the stress is given by

$$
\begin{aligned}
\boldsymbol{\sigma}(t)= & k_{\mathrm{B}} T\left[\mathrm{e}^{-\int_{0}^{t} h\left(t^{\prime}\right) \mathrm{d} t^{\prime}}\left\{\nu(0)+\int_{0}^{t} g \mathrm{e}^{\int_{0}^{t^{\prime}} k\left(t^{\prime \prime}\right) \mathrm{d} t^{\prime \prime}} \mathrm{d} t^{\prime}\right\}\right] \\
& \times\left[\int_{-\infty}^{t} \frac{\mathrm{e}^{-\left(t-t^{\prime}\right) / \tau_{0}}}{\tau_{0}} \boldsymbol{\gamma}\left(t, t^{\prime}\right) \cdot \boldsymbol{\gamma}^{+}\left(t, t^{\prime}\right) \mathrm{d} t^{\prime}\right]-P \boldsymbol{1}
\end{aligned}
$$

When $\boldsymbol{\gamma}(t)$ is given, $\boldsymbol{\alpha}(t)$ is determined by eq $11, k(t)$ by eq 9 and $\nu(t)$ by eq 13 .

\section{APPLICATION}

\section{Non-Newtonian Viscosity}

We consider the stationary shear flow with 
constant shear rate $\dot{\gamma}$. In this case

$$
\gamma(t)=\left(\begin{array}{ccc}
1 & \dot{\gamma} t & 0 \\
0 & 1 & 0 \\
0 & 0 & 1
\end{array}\right)
$$

$\boldsymbol{\alpha}$ and $\nu$ are given by

$$
\begin{aligned}
& \alpha=\left(\begin{array}{ccc}
1+2 \tau_{0}{ }^{2} \dot{\gamma}^{2} & \tau_{0} \dot{\gamma} & 0 \\
\tau_{0} \dot{\gamma} & 1 & 0 \\
0 & 0 & 1
\end{array}\right) \\
& \nu=\nu_{e} /\left(1+\frac{2}{3} \tau_{0}{ }^{2} \dot{\gamma}^{2}\right)^{1 / 2}
\end{aligned}
$$

respectively. The shear viscosity coefficient $\eta(\dot{\gamma})=\sigma_{12} / \dot{\gamma}$ and the first normal stress coefficient $\theta(\dot{\gamma})=\Delta \sigma_{n 1} / \dot{\gamma}^{2}=\left(\sigma_{11}-\sigma_{22}\right) / \dot{\gamma}^{2}$ are given as,

$$
\begin{aligned}
& \eta(\dot{\gamma})=\frac{\nu_{e} k_{\mathrm{B}} T \tau_{0}}{\left(1+\frac{2}{3} \tau_{0}{ }^{2} \dot{\gamma}^{2}\right)^{1 / 2}} \\
& \theta(\dot{\gamma})=\frac{2 \nu_{e} k_{\mathrm{B}} T_{\tau_{0}}{ }^{2}}{\left(1+\frac{2}{3} \tau_{0}{ }^{2} \dot{\gamma}^{2}\right)^{1 / 2}}
\end{aligned}
$$

Both $\eta(\dot{\gamma})$ and $\theta(\dot{\gamma})$ are the decreasing function of $\dot{\gamma}$. On the other hand, within the linear ranges of this theory in which the change in the number of chains with time is not taken into account, the absolute value of the complex viscosity $\left|\eta^{*}(i \omega)\right|$ is given by

$$
\left|\eta^{*}(i \omega)\right|=\frac{\nu_{e} k_{\mathrm{B}} T \tau_{0}}{\left(1+\tau_{0}{ }^{2} \omega^{2}\right)^{1 / 2}}
$$

where $\omega$ is the angular frequency. Equations 18 and 20 show that the shear rate dependence for $\eta(\dot{\gamma})$ and the frequency dependence for $\left|\eta^{*}(i \omega)\right|$ have almost the same functional form with respect to $\dot{\gamma}$ and $\omega$. The limiting slopes of $\log \eta(\dot{\gamma})$ vs. $\log \dot{\gamma}$ and $\log \left|\eta^{*}(i \omega)\right| v s . \quad \log \omega$ plots for high shear rates and high frequencies are 1 , this being somewhat larger than the experimental value $0.82 .^{29}$

When we define the steady state compliance in non-Newtonian flow regions by

$$
J_{\mathrm{e}}(\dot{\gamma})=\frac{\Delta \sigma_{n 1}}{2 \sigma_{12}}=\frac{\theta(\dot{\gamma})}{2 \eta(\dot{\gamma})^{2}}=\frac{\left(1+\frac{2}{3} \tau_{0}^{2} \dot{\gamma}^{2}\right)^{1 / 2}}{\nu_{e} k_{\mathrm{B}} T \tau_{0}}
$$

$J_{\mathrm{e}}(\dot{\gamma})$ is the increasing function of $\dot{\gamma}$.

Experimental evidence shows that the viscosity

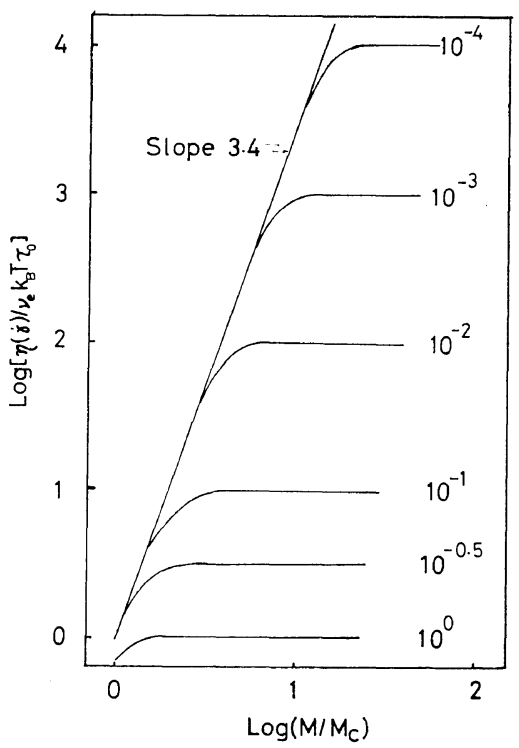

Figure 1. Logarithmic plots of shear viscosity against molecular weight in non-Newtonian flow. The number on each curve indicates the value of the parameter $\sqrt{2 / 3} \tau_{\mathrm{c}} \dot{\gamma}$. The slope of the line for Newtonian flow is 3.4. The deviation from Newtonian flow increases with an increase in the shear rate.

at the zero shear rate is proportional to $M^{3.4}$, $M$ being the molecular weight, and that $\tau_{0}$ is also proportional to $M^{3.4}$. Therefore, if we assume the relation $\tau_{0}=\tau_{\mathrm{c}}\left(M / M_{\mathrm{c}}\right)^{3.4}, \tau_{\mathrm{c}}$ being a constant and $M_{\mathrm{c}}$ the molecular weight above which the empirical 3.4th power law of viscosity is valid, we obtain the molecular weight dependence of $\eta(\dot{\gamma})$ for $\dot{\gamma}$ as shown in Figure 1, which shows a similar features as that obtained from experiments. ${ }^{29}$

Stress Growth at the Onset of the Steady Shear Flow

When the shear flow with a constant shear $\dot{\gamma}$ starts at $t=0, \gamma$ is given by eq 15 for $t>0$ and $\gamma=1$ for $t<0$. In this case $\boldsymbol{\alpha}(t)$ is obtained as

$$
\boldsymbol{\alpha}(t)=\left(\begin{array}{ccc}
1+2 \tau_{0}{ }^{2} \dot{\gamma}^{2}\{1 & & \\
\left.-\mathrm{e}^{-t / \tau_{0}}\left(1+\left(t / \tau_{0}\right)\right)\right\} & \tau_{0} \dot{\gamma}\left(1-\mathrm{e}^{-t / \tau_{0}}\right) & 0 \\
\tau_{0} \dot{\gamma}\left(1-\mathrm{e}^{-t / \tau_{0}}\right) & 1 & 0 \\
0 & 0 & 1
\end{array}\right)
$$

and $k(t)$ becomes 
$k(t)=k_{e}\left[1+\frac{2}{3} \tau_{0}^{2} \dot{\gamma}^{2}\left\{1-\mathrm{e}^{-t / \tau_{0}}\left(1+\frac{t}{\tau_{0}}\right)\right\}\right]^{1 / 2}$

Therefore, we obtain

$$
\begin{gathered}
\sigma_{12}(t)=\nu_{e} k_{\mathrm{B}} T \tau_{0} \dot{\gamma} F_{0}(t)\left(1-\mathrm{e}^{-t / \tau_{0}}\right) \\
\Delta \sigma_{n 1}(t)=2 \nu_{e} k_{\mathrm{B}} T \tau_{0}^{2} \dot{\gamma}^{2} F_{0}(t)\left\{1-\mathrm{e}^{-t / \tau_{0}}\left(1+\frac{t}{\tau_{0}}\right)\right\} \\
F_{0}(t)=\mathrm{e}^{\left.-\int_{0}^{t} k t^{\prime}\right) \mathrm{d} t^{\prime}}\left[1+k_{e} \int_{0}^{t} \mathrm{e}^{\left.\int_{0}^{t^{\prime} k\left(t^{\prime \prime}\right) \mathrm{d} t^{\prime \prime}} \mathrm{d} t^{\prime}\right]}\right.
\end{gathered}
$$

The ratios $\sigma_{12}(\dot{\gamma}, t) / \sigma_{12}(\dot{\gamma}, \infty)$ and $\Delta \sigma_{n 1}(\dot{\gamma}, t) / \Delta \sigma_{n 1}(\dot{\gamma}$, $\infty)$ are plotted against $t / \tau_{0}$ in Figure 2. The solid curves indicate the shear stress and the broken curves with dots, the normal stress.

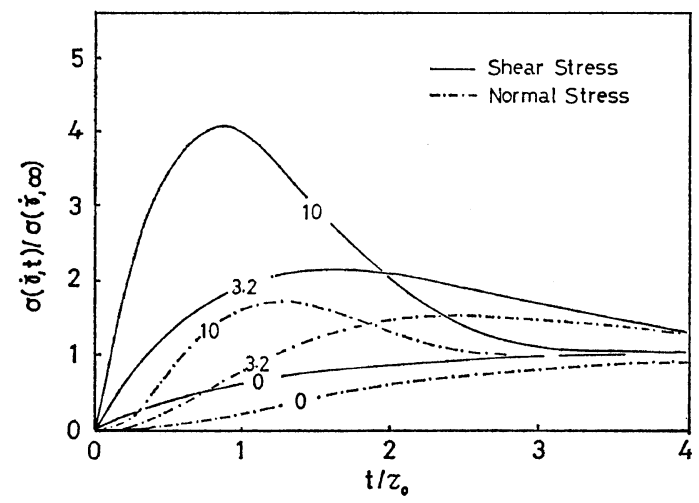

Figure 2. Stress growth at the onset of the shear flow is plotted against time. The number on each curve indicates the value of the parameter $\sqrt{2 / 3} \tau_{0} \dot{\gamma}$. The stress oveshoot appears clearly as the shear rate increases. The time required to reach the maximum value of the normal stress is almost twice as long as that of the shear stress.

The number on each curve indicates the parameter $\sqrt{2 / 3} \tau_{0} \dot{\gamma}$. The value of $k_{e} \tau_{0}$ is chosen as 0.2 for convenience in drawing the curves since $k_{e}$ is considered to be of the same order of $1 / \tau_{0}$, if the molecular chains are not very long. The stress overshoot can be seen very easily as the shear rate increases. The time needed to reach the maximum value of the normal stress is almost twice as large as that required for the shear stress. This qualitatively agrees with experimental results. Furthermore, the maximum value of $\sigma_{12}(\dot{\gamma}, t) / \sigma_{12}(\dot{\gamma}, \infty)$ becomes smaller with an increase in $k_{e} \tau_{0}$; namely, the stress overshoot is not so evident when $k_{e} \tau_{0}$ is very large. This case corresponds to the very long molecular chain system. Because, $k_{e}$ may almost be in inverse proportion to the molecular weight $M$ since the chain breakage is mainly induced by the terminal chains which decrease with increasing molecular weight and $\tau_{0}$ is proportional to $M^{3.4}$, and thus $k_{e} \tau_{0}$ increases proportionally to $M^{2,4}$.

\section{Stress Relaxation under Large Deformation}

When a constant shear deformation $\gamma_{0}$ is given, $\boldsymbol{\gamma}(t)$ is expressed as,

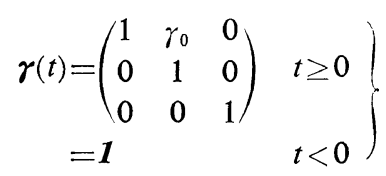

and $\boldsymbol{\alpha}(t)$ and $k(t)$ are given from eq 11 and 9 as,

$$
\begin{gathered}
\boldsymbol{\alpha}(t)=\left(\begin{array}{ccc}
1+\gamma_{0}{ }^{2} \mathrm{e}^{-t / \tau_{0}} & \gamma_{0} \mathrm{e}^{-t / \tau_{0}} & 0 \\
\gamma_{0} \mathrm{e}^{-t / \tau_{0}} & 1 & 0 \\
0 & 0 & 1
\end{array}\right) \\
k(t)=k_{e}\left(1+\frac{1}{3} \gamma_{0}{ }^{2} \mathrm{e}^{-t / \tau_{0}}\right)^{1 / 2}
\end{gathered}
$$

Therefore, the relaxation modulus $G\left(\gamma_{0}, t\right)=$ $\sigma_{12}\left(\gamma_{0}, t\right) / \gamma_{0}$ is given by

$$
G\left(\gamma_{0}, t\right)=\nu_{e} k_{\mathrm{B}} T F_{1}(t) \mathrm{e}^{-t / \tau_{0}}
$$

$F_{1}(t)=\mathrm{e}^{-\int_{0}^{t} k\left(t^{\prime}\right) \mathrm{d} t^{\prime}}\left[1+k_{\epsilon} \int_{0}^{t} \mathrm{e}^{\int_{0}^{t^{\prime} k\left(t^{\prime \prime}\right) \mathrm{d} t^{\prime \prime}}} \mathrm{d} t^{\prime}\right]$

where $k(t)$ in eq 31 is on the right hand side of eq 29.

The normal stress relaxation modulus $\Delta \sigma_{n 1}\left(\gamma_{0}\right.$, $t) / \gamma_{0}{ }^{2}$ is also given by the righthand side of eq 30 .

The relaxation modulus versus time relationship are given in Figure 3 by a log-log plot, and the number on each curve indicates the value of the parameter $\sqrt{1 / 3} \gamma_{0}$ and $k_{e} \tau_{0}$ has also been chosen here as 0.2 . The nonlinearity appears in a short period of time and the linear relation within a longer period of time. For very short periods of time not show in the figure, the linear relation is also predicted. This result agrees with the experimental results qualitatively but not quantitatively. ${ }^{25,26}$ since the experiments show that $G\left(\gamma_{0}, t\right)$ for a long definite period of time decreases with increasing $\gamma_{0}$, and the theoretical prediction on the contrary indicates that 
S. HAYASHI

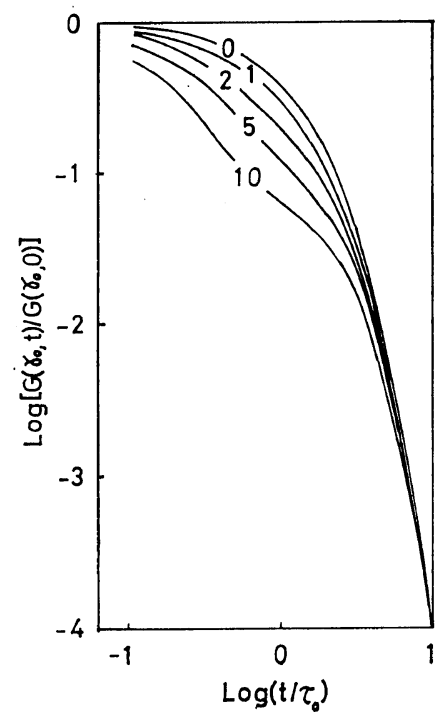

Figure 3. Logarithmic plots of relaxation modili against time. The number of each curve means the value of the parameter $\sqrt{1 / 3} \gamma_{0}$. Nonlinearity appears in a short time range and the linear relaxation tendency in a long time range.

it has nearly the same value irrespectively of the value of $\gamma_{0}$.

This disagreement between the theoretical prediction and the experimental results may be traced to the assumption that all the chains reformed at a given time have the same internal strain $\boldsymbol{\alpha}(t)$ irrespectively at the time when the chains are reformed. If the broken chains are reformed in their stress free state, the relaxation modulus at a certain time may be characterized only by those chains kept unbroken till that time. The number of chains unbroke till this time becomes smaller with increasing $\gamma_{0}$ since the chain breakage coefficient increases with $\gamma_{0}$ and therefore even for a long period of time, the relaxation modulus for a larger $\gamma_{0}$ value has a smaller value than it does for a smaller $\gamma_{0}$ value.

Stress Relaxation at a Sudden Termination of Flow

We should now like to consider the case in which a steady shear flow with constant shear rate $\dot{\gamma}$ is suddenly stopped at $t=0$. In this case $\gamma(t)$ is given by

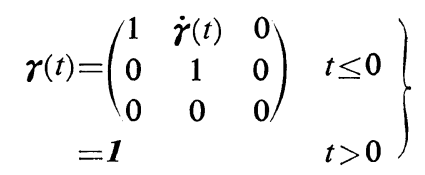

and $\boldsymbol{\alpha}(t)$ and $k(t)$ are given from eq 11 and 9 as

$$
\begin{gathered}
\boldsymbol{\alpha}(t)=\left(\begin{array}{ccc}
1+2 \tau_{0}^{2} \dot{\gamma}^{2} \mathrm{e}^{-t / \tau_{0}} & \tau_{0} \dot{\gamma} \mathrm{e}^{-t / \tau_{0}} & 0 \\
\tau_{0} \dot{\gamma} \mathrm{e}^{-t / \tau_{0}} & 1 & 0 \\
0 & 0 & 1
\end{array}\right) \\
k(t)=k_{e}\left(1+\frac{2}{3} \tau_{0}^{2} \dot{\gamma}^{2} \mathrm{e}^{-t / \tau_{0}}\right)^{1 / 2}
\end{gathered}
$$

Then $\sigma_{12}(\dot{\gamma}, t)$ and $\Delta \sigma_{n 1}(\dot{\gamma}, t)$ are given from eq 14 as

$$
\begin{gathered}
\sigma_{12}(\dot{\gamma}, t)=\nu_{e} k_{\mathrm{B}} T \tau_{0} \dot{\gamma} F_{2}(t) \mathrm{e}^{-t / \tau_{0}} \\
\Delta \sigma_{n 1}(\dot{\gamma}, t)=2 \nu_{e} k_{\mathrm{B}} T \tau_{0} \dot{\gamma}^{2} F_{2}(t) \mathrm{e}^{-t / s_{0}} \\
F_{2}(t)=\mathrm{e}^{-\int_{0}^{t} k\left(t^{\prime}\right) \mathrm{d} t^{\prime}}\left[\frac{1}{\left(1+(2 / 3) \tau_{0}{ }^{2} \dot{\gamma}^{2}\right)^{1 / 2}}\right. \\
\left.+k_{e} \int_{0}^{t} \mathrm{e}^{\int_{0}^{t^{\prime} k\left(t^{\prime \prime}\right) \mathrm{d} t^{\prime \prime}} \mathrm{d} t^{\prime}}\right]
\end{gathered}
$$

The shear stress and the normal stress show the same time dependency. The experimental evidence contrary to our prediction shows, however, that the normal stress decays more slowly

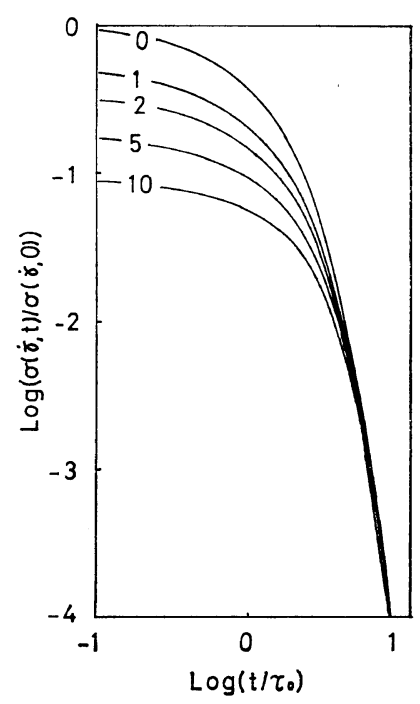

Figure 4. Logarithmic plots of shear stress after the termination of shear flow against time. The number on each curve indicates the value of the parameter $\sqrt{2 / 3} \tau_{0} \dot{\gamma}$. It shows the slower relaxation for higher shear rate in the earlier relaxation stages. 
than the shear stress. This disagreement between the theoretical and experimental results may be due to the assumption that the slip process is characterized by a single relaxation time. For a broad distribution of relaxation time, the normal stress may decay more slowly than the shear stress. Because, the normal stress and the shear stress relaxation modili contain the factors $\tau^{2}$ and $\tau$ in the integral with respect to $\tau$, respectively, the contribution from large $\tau$ is thus greater in the normal stress than in the shear stress.

Stress versus time relationships are shown in Figure 4 by a log-log plot. The number on each curve indicates the value of the parameter $\sqrt{2 / 3} \tau_{0} \dot{\gamma}$. The stress decay proceeds monotonically and the decay is slower than the linear relaxation.

\section{RELATION BETWEEN THE PRESENT THEORY AND CONSTITUTIVE EQUATION}

In the present theory, the chain breakage coefficient $k(t)$ is considered to be a function of the invariant of the internal strain tensor, and the chain generating function is constant. However, both the chain breakage coefficient and the chain generating function may generally be functions of the invariant of the internal strain tensor. Then $k$ and $g$ will be expressed as

$$
\left.\begin{array}{l}
k(t) \equiv k\left(t \mid A_{a}(t)\right) \\
g(t) \equiv g\left(t \mid A_{a}(t)\right)
\end{array}\right\}
$$

where $A_{\alpha}(t)$ mean the invariant of the internal strain tensor.

As already mentioned, the stress is expressed as

$$
\begin{aligned}
\boldsymbol{\sigma}(t)= & k_{\mathrm{B}} T \int_{-\infty}^{t} \nu(t) \frac{\mathrm{e}^{-\left(t-t^{\prime}\right) / \tau_{0}}}{\tau_{0}} \boldsymbol{\gamma}\left(t, t^{\prime}\right) \cdot \boldsymbol{r}^{+}\left(t, t^{\prime}\right) \mathrm{d} t^{\prime} \\
& -P \boldsymbol{1}
\end{aligned}
$$

where

$$
\begin{aligned}
\nu(t) & \equiv \nu\left(t \mid A_{a}(t)\right) \\
& =\mathrm{e}^{-\int_{0}^{t} k\left(t^{\prime}\right) \mathrm{d} t^{\prime}}\left[\nu(0)+\int_{0}^{t} g\left(t^{\prime}\right) \mathrm{e}^{\int_{0}^{t^{\prime}} k\left(t^{\prime \prime}\right) \mathrm{d} t^{\prime \prime}} \mathrm{d} t^{\prime}\right]
\end{aligned}
$$

On the other hand, the integral formula of the phenomenological constitutive equation is given as

$$
\boldsymbol{\sigma}(t)=\int_{-\infty}^{t} \mu\left(t, t^{\prime} \mid s\right) \gamma\left(t, t^{\prime}\right) \cdot \boldsymbol{\gamma}^{+}\left(t, t^{\prime}\right) \mathrm{d} t^{\prime}-P \mathbf{1}
$$

where $\mu\left(t, t^{\prime} \mid s\right)$ is the memory function which in turn is a function of the strain or strain rate tensor at $t$ and/or $t^{\prime}$. Many kinds of constitutive equations can be obtined by varying the choice for the functions of the invariant of strain or strain rate tensors.

We obtain eq 39 when we choose the memory function as

$$
\mu\left(t, t^{\prime} \mid s\right)=k_{\mathbf{B}} T \nu\left(t \mid A_{a}(t)\right) \frac{\mathrm{e}^{-\left(t-t^{\prime}\right) / \tau_{0}}}{\tau_{0}}
$$

Equation 42 suggests the possibility of choosing the memory function as a function of the invariant of the internal strain tensor. Each chain, however, generally has a deformation different from any of the others, so that the internal strain in the present theory may be an average strain characterized by an average relaxation time $\tau_{0}$.

The internal strain is determined by eq 4

$$
\dot{\gamma} \cdot \boldsymbol{\gamma}^{-1} \cdot \boldsymbol{\alpha}+\boldsymbol{\alpha} \cdot \boldsymbol{\gamma}^{-1} \cdot \dot{\gamma}^{+}-\dot{\alpha}=\frac{1}{\tau_{0}}(\boldsymbol{\alpha}-\boldsymbol{1})
$$

This equation shows that $\boldsymbol{\alpha}$ is determined by the strain rate so that it may satisfy the initial condition. When we treat the stress growth at the onset of flow, the initial condition is that the strain tensor is the unit tensor at the start of flow, so that the memory function contains only the invariant of strain rate tensor. In the case of stress relaxation under a constant large deformation, the strain rate is zero but the internal initial strain is equal to the given strain on the system, so that the memory function contains the invariant of strain tensor. In the case of the stress relaxation following the termination of flow, the initial state is the steady flow state, so that the memory function contains the invariant of the strain rate tensor.

When we introduce a memory function which depends on the internal strain, we have the following constitutive equation

$$
\left.\begin{array}{l}
\boldsymbol{\sigma}(t)=\int_{-\infty}^{t} \mu\left(t, t^{\prime} \mid A_{a}(t)\right) \boldsymbol{\gamma}\left(t, t^{\prime}\right) \cdot \boldsymbol{\gamma}^{+}\left(t, t^{\prime}\right) \mathrm{d} t^{\prime}-P \boldsymbol{1} \\
\boldsymbol{\alpha}(t)=\int_{-\infty}^{t} \frac{\mathrm{e}^{-\left(t-t^{\prime}\right) / \tau_{0}}}{\tau_{0}} \boldsymbol{\gamma}\left(t, t^{\prime}\right) \cdot \boldsymbol{\gamma}^{+}\left(t, t^{\prime}\right) \mathrm{d} t^{\prime}
\end{array}\right\}
$$




\section{S. HAYASHI}

The constitutive equation in the present network theory is the case in which the memory function is chosen as the right hand side of eq 42 , and we thus have

$$
\begin{gathered}
k_{\mathrm{B}} T \nu\left(t \mid A_{a}(t)\right)=\int_{-\infty}^{t} \mu\left(t, t^{\prime} \mid A_{a}(t)\right) \mathrm{d} t^{\prime} \\
k_{\mathrm{B}} T \nu\left(t \mid A_{a}(t)\right) / \tau_{0}=\mu\left(t, t \mid A_{a}(t)\right)
\end{gathered}
$$

We define the relaxation spectrum depending on the internal strain by

$$
\mu\left(t, t^{\prime} \mid A_{a}(t)\right)=\int_{-\infty}^{\infty} H\left(\tau \mid A_{a}(t)\right) \frac{\mathrm{e}^{-\left(t-t^{\prime}\right) / \tau}}{\tau} \mathrm{d} \ln \tau
$$

then we have

$$
\begin{gathered}
\int_{-\infty}^{t} \mu\left(t, t^{\prime} \mid A_{a}(t)\right) \mathrm{d} t^{\prime}=\int_{-\infty}^{\infty} H\left(\tau \mid A_{a}(t)\right) \mathrm{d} \ln \tau \\
\mu\left(t, t \mid A_{a}(t)\right)=\int_{-\infty}^{\infty} \frac{H\left(\tau \mid A_{a}(t)\right)}{\tau} \mathrm{d} \ln \tau
\end{gathered}
$$

If the memory function is characterized by a single relaxation time $\bar{\tau}$, the relaxation spectrum is written as

$$
H\left(\tau \mid A_{a}(t)\right)=H^{0}\left(\tau_{0}, t\right) \delta(\tau-\bar{\tau})
$$

since $A_{a}(t)$ contains the parameter $\tau_{0}$, where $\delta(x)$ is the delta function. In this case we have

$$
\begin{gathered}
\int_{-\infty}^{t} \mu\left(t, t^{\prime} \mid A_{\alpha}(t)\right) \mathrm{d} t^{\prime}=H^{0}\left(\tau_{0}, t\right) \\
\mu\left(t, t \mid A_{a}(t)\right)=\frac{H^{0}\left(\tau_{0}, t\right)}{\bar{\tau}}
\end{gathered}
$$

Therefore, comparing eq 44 and 45 with eq 50 and 51 , we may choose $\bar{\tau}$ in such a way that $\bar{\tau}$ is equal to $\tau_{0}$, this means that the relaxation time defined by eq 49 is equal to the relaxation time for the slip equation though $\bar{\tau}$ is related to the nonlinear relaxation phenomena.

When the memory function involves a distribution of relaxation times, $\tau_{0}$ can not be explicitly related to the relaxation spectrum defined by eq 46 and becomes an additional parameter contained in $H\left(\tau \mid A_{a}(t)\right)$. If the memory function is determined by experiment, the left hand sides of eq 47 and 48 can be derived and in this case, $H\left(\tau \mid A_{a}(t)\right)$ and $\tau_{0}$ contained in $H\left(\tau \mid A_{a}(t)\right)$ shall be determined so that they may satisfy eq 47 and 48 .
Differentiating the stress $\sigma$ with respect to $t$ we have

$$
\begin{aligned}
\dot{\boldsymbol{\gamma}} \cdot \boldsymbol{\gamma}^{-1} \cdot \boldsymbol{\sigma} & +\boldsymbol{\sigma} \cdot \boldsymbol{\gamma}^{+-1} \cdot \dot{\boldsymbol{\gamma}}+\dot{\boldsymbol{\sigma}} \\
& =\left(\frac{1}{\tau_{0}}-\frac{\dot{\nu}}{\nu}\right) \boldsymbol{\sigma}-P\left(\dot{\boldsymbol{\gamma}}+\dot{\boldsymbol{\gamma}}^{+}\right)
\end{aligned}
$$

where we put $P=k_{\mathrm{B}} T$ for the sake of simplicity. This equation indicates that the apparent relaxation time $\tau_{\mathrm{app}}$ is expressed by

$$
\frac{1}{\tau_{\mathrm{app}}}=\frac{1}{\tau_{0}}-\frac{\dot{\nu}}{\nu}
$$

The apparent relaxation time seems longer than $\tau_{0}$ when the chains increase and shorter when they decrease.

Aknowledgment. This work has been partially supported by the Grant-in-Aid for Scientific Research from the Ministry of Education to which we should like to express our sincere appreciation.

\section{REFERENCES}

1. M. Yamamoto, J. Phys. Soc. Jpn., 11, 413 (1956); 12, 1148 (1957); 13, 1200 (1958).

2. A. S. Lodge, Trans. Faraday Soc., 52, 120 (1956).

3. K. W. Scott and R. S. Stein, J. Chem. Phys., 21, 1281 (1959).

4. S. Hayashi, Prog. Theor. Phys. Supplement, 10, 82 (1959).

5. S. Hayashi, J. Phys. Soc. Jpn., 18, 131 (1963); 19, 101 (1963); 19, 2306 (1964).

6. S. Hayashi and Y. Takano, J. Phys. Soc. Jpn., 23, 1063 (1967).

7. Y. Takano, Polym. J. 6, 61 (1974), J. Soc. Rheol. Jpn., 2, 87 (1974).

8. A. S. Lodge, Rheol. Acta., 7, 379 (1968).

9. A. S. Lodge, "Elastic Liquid," Academic Press, New York, N.Y., 1964

10. J. L. White and A. B. Mentzner, J. Polym. Sci., 7, 1867 (1963).

11. R. B. Bird, Chem. Eng. Prog. Symp., Ses. 58, 61 (1965).

12. T. W. Spriggs, J. D. Huppler and R. B. Bird, Trans. Soc. Rheol., 10, 191 (1966).

13. T. W. Spriggs, Chem. Eng. Sci., 20, 931 (1965).

14. R. B. Bird and P. J. Carrear, Chem. Eng. Sci., 23, 427 (1968).

15. L. J. Zapas and J. C. Phillips, J. Res. Natl. Bur. Stands., 75A, 93 (1971).

16. M. Yamamoto, Appl. Polym. Symp., 20, 3 (1973). 
A Network Theory of Nonlinear Viscoelasticity

17. M. Yamamoto, "Theory of Deformation of Bodies," Seibundo Shinkosha Publishing Co., Tokyo, (1972).

18. M. Yamamoto, J. Soc. Mater. Sci. Jpn., 21, 355 (1972).

19. M. Yamamoto, Trans. Soc. Rheol. 15, 331 (1971).

20. M. Takahashi, T. Masuda, and S. Onogi, $J$. Soc. Rheol. Jpn., 1, 16 (1973).

21. M. Takahashi and S. Onogi, J. Soc. Rheol. Jpn., 2, 95 (1974); 3, 1 (1975).

22. K. Osaki, M. Fukuda, S. Ota, and M. Kurata, J. Soc. Rheol. Jpn., 2, 106 (1974).

23. M. Fukuda, K. Osaki, and M. Kurata, J. Soc. Rheol. Jpn., 2, 110 (1974).
24. Y. Einaga, K. Osaki, M. Kurata, S. Kimura, and M. Tamura, Polym. J., 2, 550 (1971).

25. Y. Einaga, K. Osaki, M. Kurata, S. Kimura, N. Yamada, and M. Tamura, Polym. J., 5, 91 (1973).

26. K. Osaki, Y. Einaga, M. Kurata, N. Yamada, and M. Tamura, Polym. J., 5, 283 (1973).

27. W. P. Cox and F. H. Merz, J. Polym. Sci., 28, 619 (1958).

28. W. W. Grassley and L. Segal, Macromolecules, 2, 47 (1969).

29. R. A. Stratton, J. Collod Sci., 22, 517 (1966).

30. M. Sakai, H. Fukaya, and M. Nagasawa, Trans. Soc. Rheol., 16, 635 (1972). 